\title{
Growth during the first year in infants affected by neonatal abstinence syndrome
}

Tammy E. Corr ${ }^{1 *}$, Eric W. Schaefer ${ }^{2}$ and Ian M. Paul ${ }^{1,2}$

\begin{abstract}
Background: Infants with neonatal abstinence syndrome (NAS) initially experience neurologic excitability, poor feeding, and/or hyperphagia in the setting of increased metabolic demand. Because the longitudinal effects of these early symptoms and behaviors on weight trends are unknown, we sought to contrast weight gain patterns through age 1 year for infants diagnosed with NAS with matched controls.

Methods: Retrospective cohort of 70 singletons with a gestational age of $\geq 37$ weeks and an ICD-9 or ICD-10 diagnosis of NAS made $\leq 7$ days after birth with institutional follow-up matched to patients without NAS. Infants were matched on gestational age ( \pm 2 weeks), birth weight $( \pm 20 \mathrm{~g})$, sex (exact), and insurance type (exact). Quantile regression methods were used to estimate 10th, 25th, 50th, 75th and 90th percentiles of weight over time.
\end{abstract}

Results: The mean gestational age for an infant with NAS was 38.8 weeks (standard deviation [SD], 1.3). The mean birth weight was $3.141 \mathrm{~kg}$ (SD, 0.510). NAS patients had a median of 24 weights recorded between birth and 400 days (inter-quartile range [IQR], 16-32 weights). Patients without NAS had a median of 12 weights recorded (IQR, 10-16). Growth curves were similar over the first 400 days of life. Patients with NAS had non-significantly higher and lower estimated weights for the 90th and 10th percentiles, respectively.

Conclusion: Infants with a diagnosis of NAS grew similarly to controls during their first year. Given the frequentlyencountered NAS symptoms of hyperphagia and irritability, future studies may evaluate whether early differences in caregiver feeding exist and whether they have longer-term impacts on growth.

Keywords: Neonatal abstinence syndrome, Neonatal opioid withdrawal syndrome, Infant growth, Infant nutrition, Pediatric obesity, Behavioral feeding, Comfort feeding, Parenting practices

\section{Background}

Neonatal abstinence syndrome (NAS) is a growing public health problem both nationally and globally [1-4]. Infants affected by neonatal abstinence syndrome display a number of symptoms and behaviors related to neurologic excitability including increased tone, tremors, hyperthermia, tachypnea, excessive crying, and increased time in an awake state [5]. Additionally, these infants often exhibit poor feeding with an uncoordinated suck as well as symptoms of gastrointestinal dysfunction such as regurgitation and emesis and loose or watery stools [5]. This constellation of neurologic and gastrointestinal symptoms may result in caloric intake that is inadequate and fails to meet

\footnotetext{
*Correspondence: tcorr@pennstatehealth.psu.edu

${ }^{1}$ Penn State College of Medicine, Department of Pediatrics, P.O. Box 850, 500 University Drive, Hershey, PA 17033-0850, USA

Full list of author information is available at the end of the article
}

the increased metabolic demands of the symptomatic infant resulting in hyperphagia [6].

Studies focused on caloric intake and growth of infants affected by NAS in the immediate neonatal period are inconsistent and sparse [6-9]. While some studies suggest weight loss in the neonatal period is greater in drug-exposed infants [7,9], other studies report infants seem to compensate for this hypermetabolic state by increased intake [6], while still others propose that this hyperphagia can lead to excessive weight gain [8]. Even less is known about the feeding patterns and subsequent growth of these affected infants as they age [10].

Infants affected by NAS are symptomatically irritable and difficult to soothe. These characteristics along with early hyperphagia may lead to the development of aberrant feeding behaviors by caregivers with a tendency towards feeding to comfort. While infants have an innate

(c) The Author(s). 2018 Open Access This article is distributed under the terms of the Creative Commons Attribution 4.0 International License (http://creativecommons.org/licenses/by/4.0/), which permits unrestricted use, distribution, and 
ability to control their caloric intake [11, 12], parental feeding practices can alter eating behavior and affect subsequent weight gain and growth [13]. Therefore, we sought to estimate the weight patterns through age 1 year for infants affected by NAS compared with matched controls without NAS. We hypothesized that infants affected by NAS would have larger weight gains than those of matched controls and a tendency towards obesity.

\section{Methods \\ Design}

In this retrospective cohort study, electronic medical records (EMR) of all newborns $(N=13,718)$ hospitalized at the Penn State Milton S. Hershey Medical Center (HMC; Hershey, PA) between July 2008 and March 2016 were queried. HMC is a tertiary care center with a level IV neonatal intensive care unit (NICU) and an active maternal-fetal medicine (MFM) program. Data extracted from the EMR included birth weight, type of delivery (vaginal or Cesarean), sex, gestational age, singleton versus multiple birth, NICU stay, insurance status (public, private, self-pay), receipt of drugs (morphine, phenobarbital, clonidine) during birth hospitalization, total number of inpatient and outpatient visits within 13 months after birth, and weights and lengths entered in the EMR during inpatient or outpatient visits. Where data were missing from searchable fields of NAS patients, they were manually extracted from the clinical chart. For all transferred newborns with a diagnosis of NAS $(N=127)$, pre-transferal data were obtained by physically reviewing outside records.

\section{Participants}

Analysis was restricted to newborns of singleton birth with a gestational age $\geq 37$ weeks and a diagnosis of NAS made $\leq 7$ days after birth who had $\geq 3$ weights recorded after discharge with $\geq 1$ weight recorded between 100 and 400 days to ensure adequate follow up. NAS cases were identified using the ICD-9 diagnosis code 779.5 (drug withdrawal syndrome in a newborn) and the ICD-10 code P96.1 (neonatal withdrawal symptoms from maternal use of drugs of addiction). Each NAS patient was matched to 1 patient without NAS on gestational age ( \pm 2 weeks), birth weight $( \pm 25 \mathrm{~g}$ ), sex, and insurance (private vs. public, exact match) using a greedy matching algorithm [14]. While the acceptable range for matching on gestational age was wide, the majority were exact matches, and only 1 match had a 2-week difference. Eligible matches were well singletons with no NICU stay, a gestational age of $\geq 37$ weeks with record of birthweight who had $\geq 3$ weights recorded after discharge with $\geq 1$ weight recorded between 100 and 400 days. A total of 2900 patients without NAS met these inclusion criteria. For matching, self-pay was combined with Medicaid and missing insurance status was imputed as private.

\section{Data analysis}

We used a quantile regression model appropriate for longitudinal data to estimate 10th, 25th, 50th (median), 75th, and 90th percentiles of weight (measured in $\mathrm{kg}$ ) as a function of time after birth. The penalized fixed-effects model in the $\mathrm{R}$ package "Regression Quantiles for Panel Data (rqpd)" was used to estimate the percentile curves [15]. The model includes separate intercepts for each patient to account for the correlation among repeated weights measured for a patient. Regularization was used to estimate the intercepts with the amount of regularization controlled by a tuning parameter $(\lambda)$. Separate models were fitted for patients treated with NAS and their matched controls so that $\lambda$ could be specified differently in each model and adjusted accordingly for the smaller number of weights recorded for matched controls. The parameter $\lambda$ was set equal to 50 for NAS patients and to 20 for matched controls.

To estimate non-linear percentile curves, we used restricted cubic splines [16] with knots at each quartile of weight and a knot at 2 days after birth. The knot at 2 days was used to model the early and expected loss of weight in the first days after birth. All weights recorded from birth to 400 days after birth were used. Three obvious errors in recorded weights were deleted (e.g. a value of $0 \mathrm{~kg}$ ). Final percentile curves were only shown to 390 days (approximately 13 months after birth). Non-parametric bootstrapping with 1000 bootstrap samples was used to test for differences between NAS patients and matched controls for each percentile curve. In these bootstrap samples, matched pairs were randomly selected, and all weights from selected patients were used in respective models fits in each bootstrap sample.

We conducted 2 subgroup analyses. First, we compared percentile curves of patients who did and did not receive pharmacologic therapy for NAS. Second, we compared patients with NAS who received pharmacologic therapy to their matched controls. The same methods were used as above with the following changes: $\lambda$ was set to 20 and only 4 total knots were used (instead of 5) for patients with NAS who did not receive pharmacologic therapy due to the smaller number of weights recorded for this subgroup.

\section{Results}

Among the 234 neonates with a diagnosis of NAS and documented inpatient stay at our center, 70 (30\%) met all inclusion criteria and were included in 


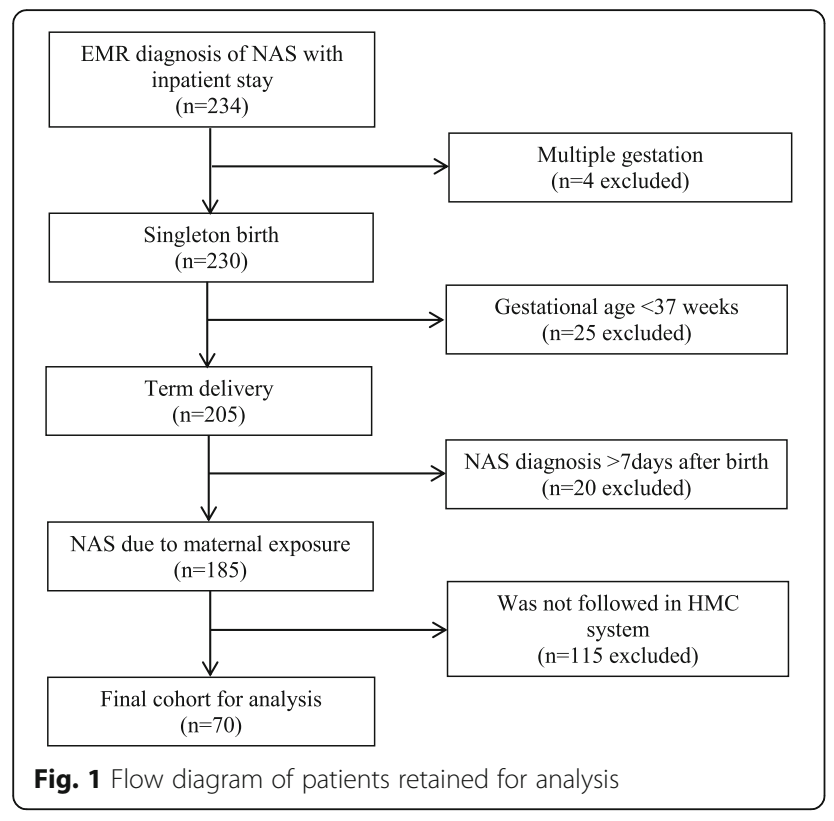

the final analysis (Fig. 1). Thirty-seven percent of the NAS population was early term, $50 \%$ term, and $13 \%$ post term. NAS patients were primarily insured by Medicaid (81\%). Similar to controls, about one third of NAS infants were born via C-section, and the majority (89\%) were inborn. Nearly $75 \%$ of NAS patients had a NICU admission. Median lengths of stay were 11.1 days for patients with NAS and 2.2 days for controls (Table 1).

Fifty $(71 \%)$ of the NAS patients were $<1$ day old at the time of NAS diagnosis. An additional $14 \%$ were diagnosed on the day following birth, and the remaining $14 \%$ of patients were diagnosed between ages $2-5$ days. Thirty-six (51\%) of NAS patients did not receive pharmacologic treatment. Among NAS patients who required pharmacologic therapy, nearly all (97\%) received treatment with morphine with 4 patients (12\%) requiring the addition of a second agent (phenobarbital or clonidine) to manage their symptoms. One patient received treatment with phenobarbital alone.

Table 1 Demographic and birth characteristic of infants with NAS and matched controls

\begin{tabular}{|c|c|c|c|}
\hline \multirow[t]{2}{*}{ Variable } & NAS & \multirow{2}{*}{$\begin{array}{l}\text { Matched Controls } \\
(n=70)\end{array}$} & \\
\hline & $(n=70)$ & & \\
\hline \multicolumn{4}{|l|}{$\overline{S e x^{b}}$} \\
\hline Male & $30(43 \%)$ & $30(42.9 \%)$ & \\
\hline Female & $40(57 \%)$ & $40(57.1 \%)$ & \\
\hline \multicolumn{4}{|l|}{ Gestational age (weeks) ${ }^{b}$} \\
\hline 37 & $13(19 \%)$ & $13(19 \%)$ & \\
\hline 38 & $13(19 \%)$ & $12(17 \%)$ & \\
\hline 39 & $25(36 \%)$ & $28(40 \%)$ & \\
\hline 40 & $10(14 \%)$ & $10(14 \%)$ & \\
\hline 41 & $9(13 \%)$ & $7(10 \%)$ & \\
\hline \multicolumn{4}{|l|}{ Birth weight $(\mathrm{kg})^{\mathrm{b}}$} \\
\hline Median & 3.030 & 3.033 & \\
\hline (Interquartile range) & $2.746-3.465$ & $2.750-3.465$ & \\
\hline \multicolumn{4}{|l|}{ Insurance ${ }^{b}$} \\
\hline Private & $13(19 \%)$ & $13(19 \%)$ & \\
\hline \multirow[t]{2}{*}{ Medicaid/self-pay } & $57(81 \%)$ & $57(81 \%)$ & \\
\hline & & & $P$-value \\
\hline Transferred from outside hospital & $8(11 \%)$ & $0(0 \%)$ & 0.003 \\
\hline Type of delivery & & & 0.990 \\
\hline Vaginal & $48(69 \%)$ & $47(67 \%)$ & \\
\hline Cesarean & $22(31 \%)$ & $23(33 \%)$ & \\
\hline NICU ${ }^{c}$ stay & $52(74.3 \%)$ & $0(0 \%)$ & $N / A^{d}$ \\
\hline Total length of stay (days) & & & $<0.001$ \\
\hline Median (Interquartile range) & $11.1(5.3-22.3)$ & $2.2(1.9-2.6)$ & \\
\hline
\end{tabular}

$N A S^{a}$, Neonatal Abstinence Syndrome; ${ }^{b}$ matched characteristic; $N I C U^{c}$, Neonatal Intensive Care Unit; $N / A^{d}$, not applicable as controls were required to have no NICU stay; thus, the groups are different by definition 
Patients with NAS had a total of 2072 weights recorded between birth and 400 days of age (median = 24, inter-quartile range 16-32) compared to 974 weights recorded (median $=12$, inter-quartile range 10-16) during the same time period for matched controls. The majority (61\%) of the weights for patients with NAS were recorded during the initial birth hospitalization with a median of 12 weights recorded during the birth hospitalization and a median of 9 weights recorded between newborn discharge and 400 days. In contrast, for matched controls, the median number of weights recorded during the birth hospitalization was 3 , and a median of 10 weights were recorded between newborn discharge and 400 days.

Figure 2 displays individual growth trajectories of patients with NAS and their matched counterparts, while Fig. 3 shows the estimated percentile curves of weight as a function of time after birth for NAS patients and matched controls. Percentile estimates were generally similar between groups, although the 10th and 90th percentiles were wider for NAS patients. However, no differences were statistically significant between groups for any percentile.

In a subgroup analysis, Fig. 4 shows the percentile curves of patients with a diagnosis of NAS who received pharmacologic therapy $(N=34)$ and those who did not $(N$ $=36$ ). Differences between groups were non-significant for each percentile. In a separate subgroup analysis, Fig. 5 shows percentile curves of patients with NAS requiring pharmacologic therapy and matched controls. Differences were again non-significant.

\section{Discussion}

This retrospective, pilot analysis of data from a single center failed to reveal significant growth differences between birth and 1 year among those infants diagnosed with NAS when compared with matched controls. Further subgroup analysis of those NAS infants pharmacologically treated compared to matched controls did not demonstrate growth differences. These results conflict with our a priori hypotheses, which reflected known feeding difficulties and hyperphagia among infants with NAS.

Pediatric growth is a complex, multifactorial process influenced by genes, nutritional intake, the environment, overall health, and socioeconomic status (SES). In the newborn period, the NAS population is unique in its nutritional needs. The hypermetabolic state resulting from symptoms of withdrawal in combination with poor feeding places this patient population at risk for excessive weight loss in the neonatal period $[7,9]$. While the neonate may compensate for this hypermetabolic state by increased intake [6], there is some evidence that these eating habits may lead to undue weight gain [8]. Our study failed to support either of these patterns of growth.

Instead, consistent with a previous investigation by Vance et al. [10], we found similar weight gain trends between infants affected by NAS and their matched counterparts. This likeness existed when comparing all infants with a diagnosis of NAS to matched controls and when comparing controls only to NAS patients with more severe disease who were treated with pharmacologic therapy. Reasons for this lack of difference may be due to our small sample size of just 70 patients. Indeed, there appears to be a trend, albeit non-significant, towards NAS patients having higher estimated weight values for the 90th percentile and smaller estimated values for the 10th percentile.

It is reasonable to presume differences may exist in the growth of this population for a number of reasons. Similar to previous study findings $[3,17]$, NAS patients cared for at our center were predominantly insured by Medicaid, a proxy for lower socioeconomic status [18]. There is an abundance of data that suggest children
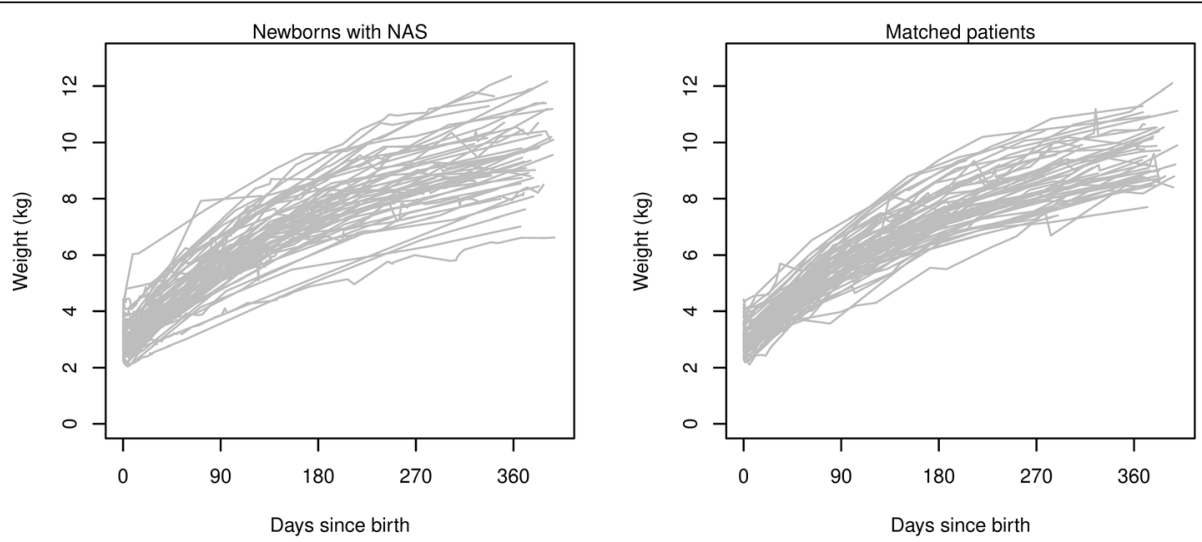

Fig. 2 Individual growth trajectories of weight for patients with neonatal abstinence syndrome (left) and matched comparison patients (right) show similar growth patterns 


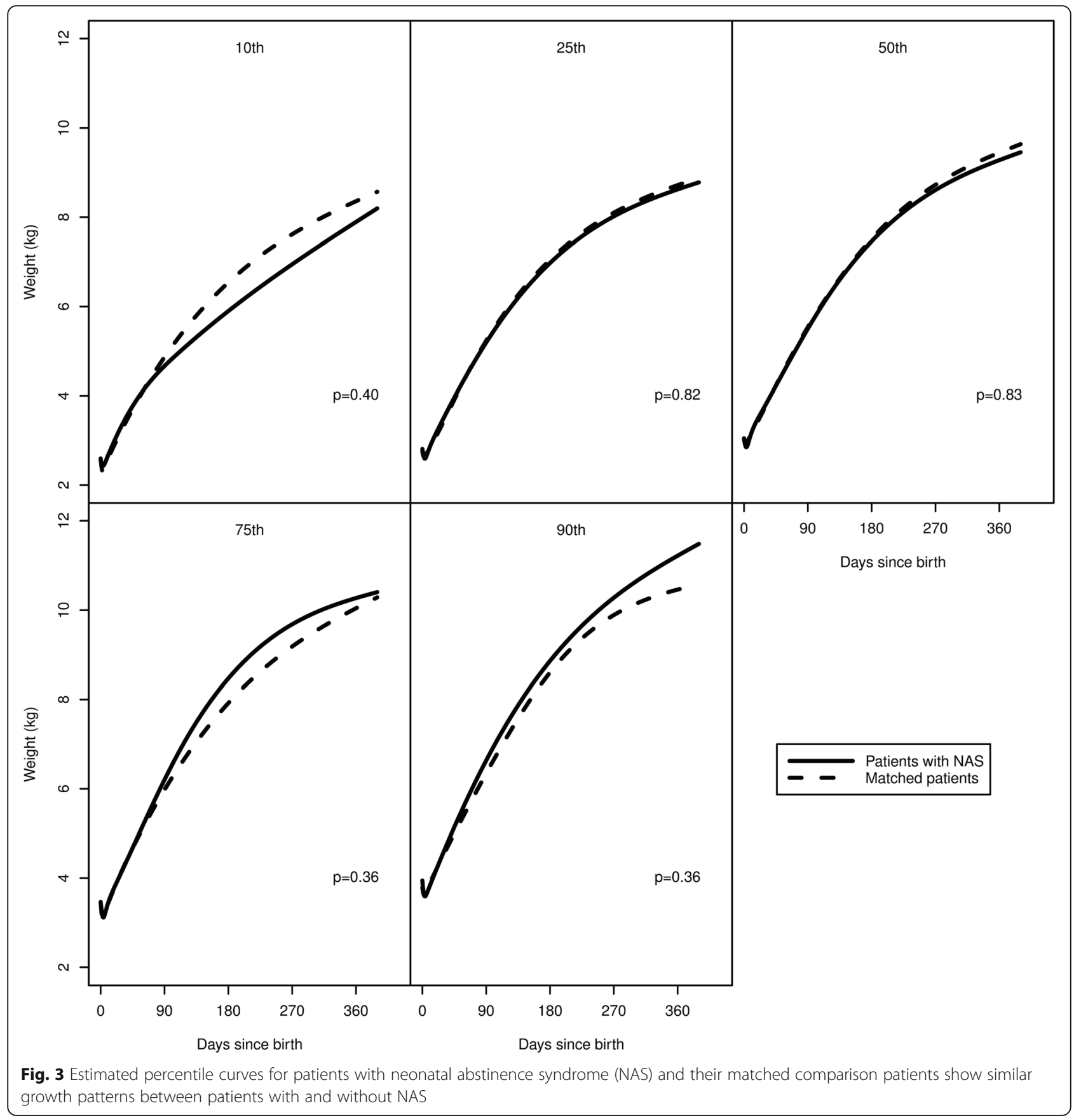

affected by poverty are at risk for abnormal weight gain. Wright et al. revealed that children of deprivation were 2.2 times more likely than children with adequate resources to have failure to thrive [19], and more recent data from developing countries suggest children from low-income households are at risk for both undernutrition and overnutrition [20,21]. In developed countries such as the United States, there are numerous studies that indicate there is an inverse relationship between weight and SES [22-24].
However, the burden of NAS is experienced by members of all socioeconomic statuses, and deprivation alone is not the only reason to suspect variance in growth. There is compelling evidence to suggest early feeding behaviors affect childhood eating habits and weight $[25,26]$. Hyperphagia and significant irritability are characteristic symptoms in newborns affected by NAS. In an effort to soothe these agitated infants, caregivers may feed to comfort under the incorrect assumption the infant is crying 


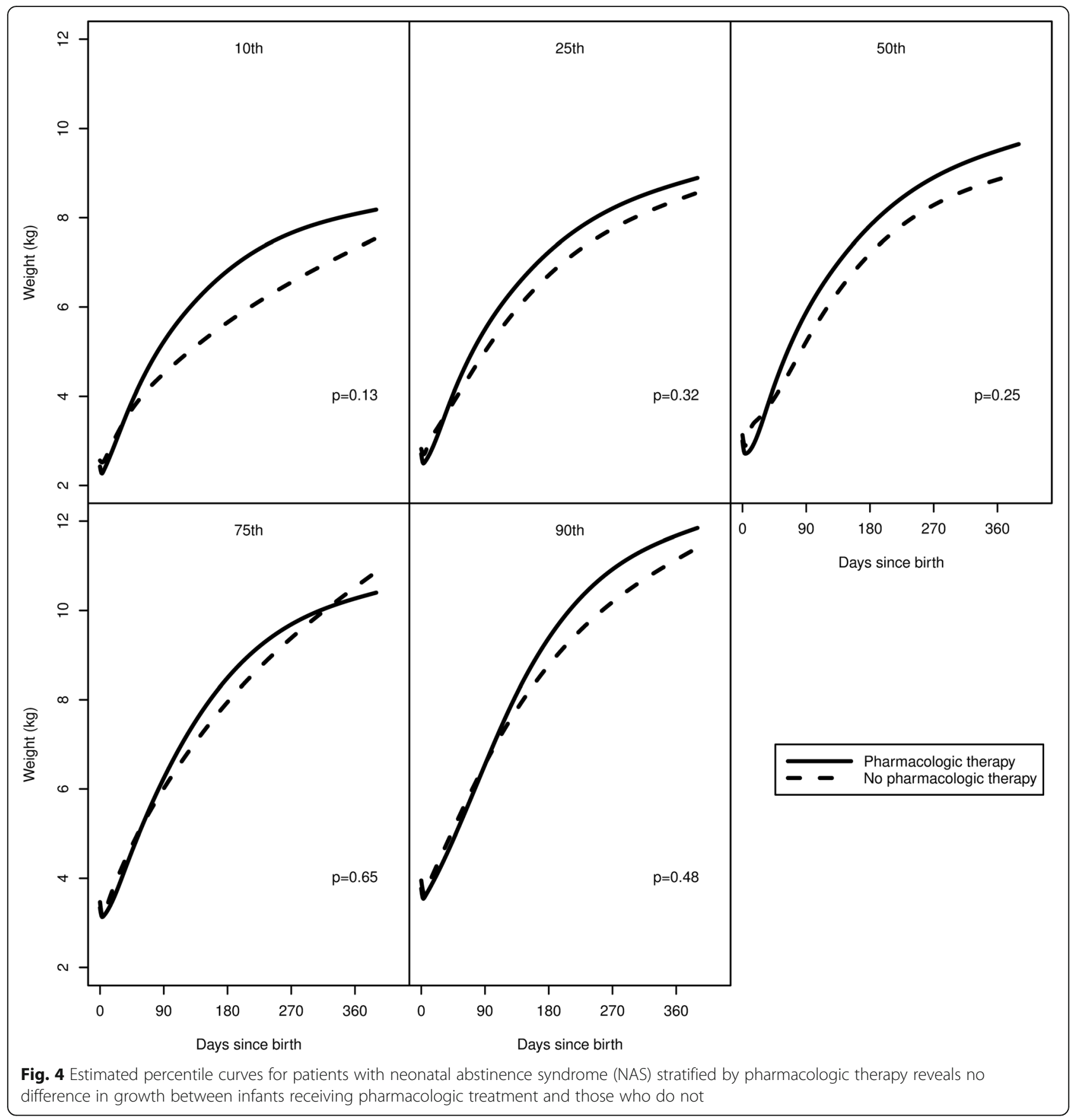

secondary to hunger. While infants have an innate ability to control their caloric intake [11, 12], parental feeding practices can alter eating behavior and affect subsequent weight gain and growth [13]. Therefore, it is reasonable to suspect that this population is at risk for development of abnormal feeding behaviors with a consequent tendency towards obesity. It is also equally plausible to presume this behavior is modifiable as recently demonstrated in the INSIGHT trial with infants not affected by NAS [27].
There are a number of limitations to our study. Our data are retrospective and gathered from a single center with a fairly homogenous population. Many patients ultimately received their post-discharge care outside the HMC system, and the resulting sample size is small and may conceal actual differences that exist in growth of this vulnerable population. Additionally, utilizing a hospital database for research depends on correct ICD coding. Failing to assign the relevant diagnostic code for an infant who displays symptoms of NAS may lead to 


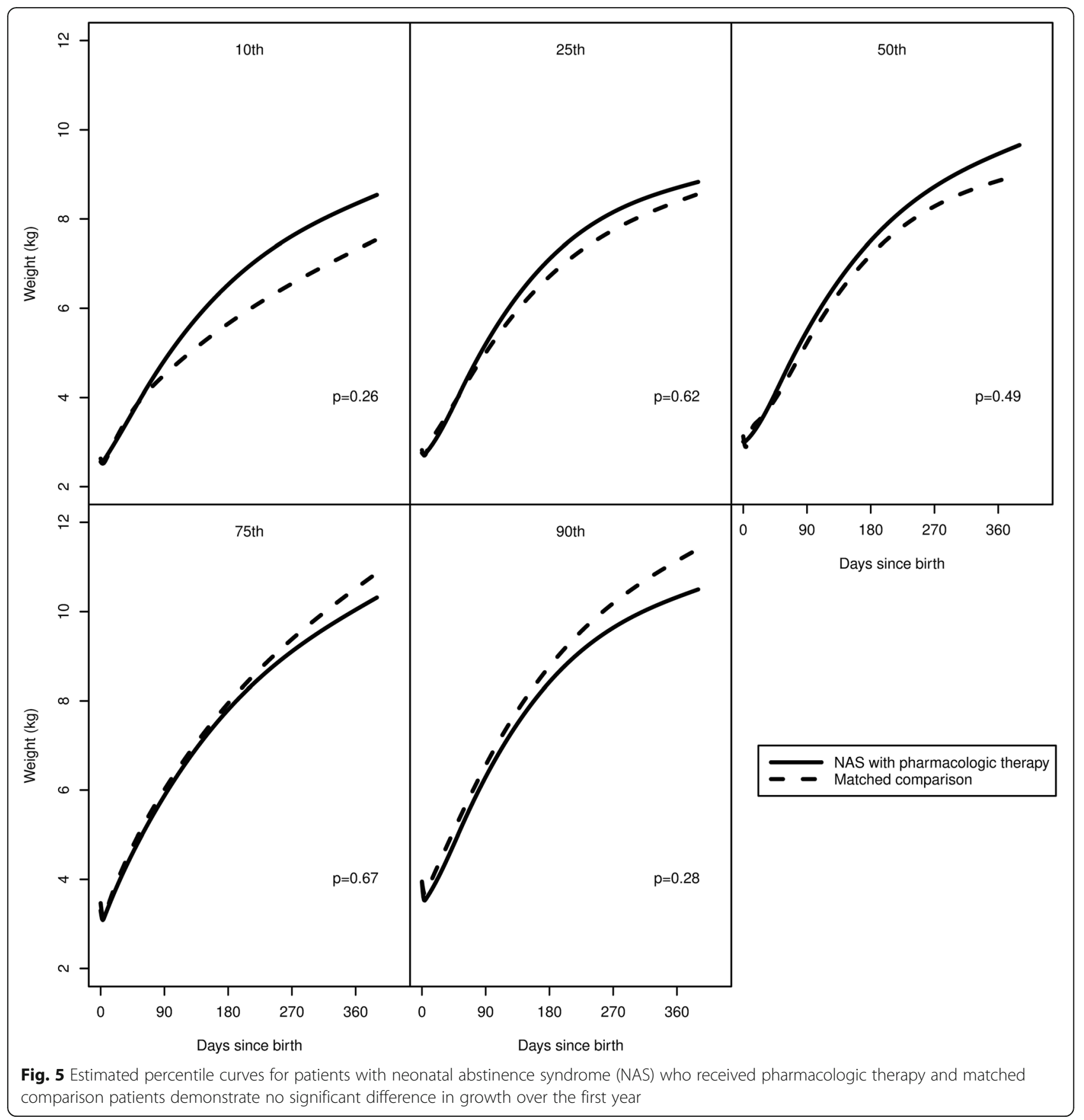

a falsely-low appreciation of the true extent of this syndrome at our institution. Conversely, inappropriately assigning a diagnosis of NAS to an infant being observed for NAS may lead to improper selection of the desired study population. Indeed, in our dataset, over half of the patients with a diagnosis of NAS did not receive pharmacologic management, suggesting that their symptoms were mild or they were inaccurately assigned such a diagnosis. Future research using a larger database with access to long-term follow-up data may clarify whether differences in growth exist between patients affected by NAS and their non-affected counterparts.

\section{Conclusion}

Infants with a diagnosis of NAS grew similarly to matched controls in this small, retrospective sample from a single center. Future studies may evaluate whether early differences in caregiver feeding exist, and if so, whether they have longer-term impacts on growth of these infants. 


\section{Abbreviations}

EMR: Electronic medical records; HMC: Hershey Medical Center; IQR: Interquartile range; MFM: Maternal fetal medicine; NAS: Neonatal abstinence syndrome; NICU: Neonatal intensive care unit; SD: Standard deviation; SES: Socioeconomic status

\section{Acknowledgements}

The authors would like to acknowledge Jessica Beiler of the Penn State Health Pediatric Clinical Research Office for her assistance in data collection.

\section{Funding}

The first author received funding through the Penn State Children's Hospital, Children's Miracle Network to support the work completed in this study. They played no role in the design of the study or collection, analysis, and interpretation of data.

\section{Availability of data and materials}

The datasets used and/or analysed during the current study are available from the corresponding author on reasonable request.

\section{Authors' contributions}

TC contributed to the conception and design of the study, interpretation of data, drafting the manuscript and approving the final version. ES contributed to the design of the study; acquisition, analysis, and interpretation of the data; drafting of the manuscript; and approving of the final version. IP contributed to the conception and design of the study, interpretation of data, editing of the manuscript, and approving of the final version of the manuscript.

\section{Ethics approval and consent to participate}

Approval for this study was obtained by the Penn State Hershey Institutional Review Board under the reference number, STUDY00005068. Because patient information was deidentified, electronic health data, for the purposes of our study, consent was not obtained.

\section{Consent for publication}

Not applicable. Data was collected and de-identified for the purposes of analysis.

\section{Competing interests}

The authors declare that they have no competing interests.

\section{Publisher's Note}

Springer Nature remains neutral with regard to jurisdictional claims in published maps and institutional affiliations.

\section{Author details}

${ }^{1}$ Penn State College of Medicine, Department of Pediatrics, P.O. Box 850, 500 University Drive, Hershey, PA 17033-0850, USA. ${ }^{2}$ Penn State College of Medicine, Department of Public Health Sciences, Hershey, PA, USA.

Received: 6 March 2018 Accepted: 25 October 2018

Published online: 05 November 2018

\section{References}

1. Patrick SW, Davis MM, Lehmann CU, Cooper WO. Increasing incidence and geographic distribution of neonatal abstinence syndrome: United States 2009 to 2012. J Perinatol. 2015;35(8):650-5.

2. Tolia VN, Patrick SW, Bennett MM, Murthy K, Sousa J, Smith PB, et al. Increasing incidence of the neonatal abstinence syndrome in U.S. neonata ICUs. NEJM. 2015;372(22):2118-26.

3. Corr TE, Hollenbeak CS. The economic burden of neonatal abstinence syndrome in the United States. Addiction. 2017;112:1590-9.

4. Kocherlakota P. Neonatal abstinence syndrome. Pediatrics. 2014;134(2): e547-61.

5. Hudak ML, Tan RC. Neonatal drug withdrawal. Pediatrics. 2012;129(2): e540-e60.

6. Martinez A, Kastner B, Taeusch HW. Hyperphagia in neonates withdrawing from methadone. Arch Dis Child Fetal Neonatal Ed. 1999;80(3):F178-82.
7. Weinberger SM, Kandall SR, Doberczak TM, Thornton JC, Bernstein J. Early weight-change patterns in neonatal abstinence. Am J Dis Child. 1986;140(8):829-32.

8. Shephard R, Greenough A, Johnson K, Gerada C. Hyperphagia, weight gain and neonatal drug withdrawal. Acta Paediatr. 2002;91(9):951-3.

9. Dryden C, Young D, Campbell N, Mactier H. Postnatal weight loss in substitute methadone-exposed infants: implications for the management of breast feeding. Arch Dis Child Fetal Neonatal Ed. 2012;97(3):F214-6.

10. Vance JC, Chant DC, Tudehope DI, Gray PH, Hayes AJ. Infants born to narcotic dependent mothers: physical growth patterns in the first 12 months of life. J Paediatr Child Health. 1997:33(6):504-8.

11. Cohen RJ, Brown KH, Canahuati J, Rivera LL, Dewey KG. Effects of age of introduction of complementary foods on infant breast milk intake, total energy intake, and growth: a randomised intervention study in Honduras. Lancet. 1994:344(8918):288-93.

12. Fomon SJ, Filer $L J J r$, Thomas LN, Rogers RR, Proksch AM. Relationship between formula concentration and rate of growth of normal infants. J Nutr. 1969;98(2):241-54.

13. Paul IM, Bartok CJ, Downs DS, Stifter CA, Ventura AK, Birch LL. Opportunities for the primary prevention of obesity during infancy. Adv Pediatr Infect Dis. 2009;56:107-33.

14. Bergstralh DJKJ. Computerized matching of controls. Mayo Clinic: Rochester; 1995.

15. Koenker R. Quantile regression for longitudinal data. JMVA. 2004:91(1):74-89.

16. Koenker R, Ng PIN, Portnoy S. Quantile smoothing splines. Biometrika. 1994; 81(4):673-80.

17. Patrick SW, Schumacher RE, Benneyworth BD, Krans EE, McAllister JM, Davis MM. Neonatal abstinence syndrome and associated health care expenditures. JAMA. 2012;307(18):1934-40.

18. Rosenbaum S. Medicaid. NEJM. 2002;346(8):635-40.

19. Wright CM, Waterston A, Aynsley-Green A. Effect of deprivation on weight gain in infancy. Acta Paediatr. 1994;83(4):357-9.

20. Abdullah $\mathrm{A}$. The double burden of undernutrition and overnutrition in developing countries: an update. Curr Obes Rep. 2015;4(3):337-49.

21. Tzioumis E, Adair LS. Childhood dual burden of under- and overnutrition in low- and middle-income countries: a critical review. Food Nutr Bull. 2014; 35(2):230-43.

22. Barriuso L, Miqueleiz E, Albaladejo R, Villanueva R, Santos JM, Regidor E. Socioeconomic position and childhood-adolescent weight status in rich countries: a systematic review, 1990-2013. BMC Pediatr. 2015;15:129.

23. Wang $Y$, Lim H. The global childhood obesity epidemic and the association between socio-economic status and childhood obesity. Int Rev Psychiatry. 2012;24(3):176-88.

24. Wu S, Ding Y, Wu F, Li R, Hu Y, Hou J, et al. Socio-economic position as an intervention against overweight and obesity in children: a systematic review and meta-analysis. Sci Rep. 2015:5:11354.

25. Davison KK, Birch LL. Childhood overweight: a contextual model and recommendations for future research. Obes Rev. 2001:2(3):159-71.

26. Savage JS, Fisher JO, Birch LL. Parental influence on eating behavior: conception to adolescence. J Law Med Ethics. 2007:35(1):22-34.

27. Savage JS, Birch LL, Marini M, Anzman-Frasca S, Paul IM. Effect of the INSIGHT responsive parenting intervention on rapid infant weight gain and overweight status at age 1 year: a randomized clinical trial. JAMA Pediatr. 2016;170(8):742-9.

Ready to submit your research? Choose BMC and benefit from:

- fast, convenient online submission

- thorough peer review by experienced researchers in your field

- rapid publication on acceptance

- support for research data, including large and complex data types

- gold Open Access which fosters wider collaboration and increased citations

- maximum visibility for your research: over $100 \mathrm{M}$ website views per year

At $\mathrm{BMC}$, research is always in progress.

Learn more biomedcentral.com/submission 